\title{
Impact of problem context on students' concept definition of an expectation value
}

\author{
Benjamin P. Schermerhorn $\odot,{ }^{1,2}$ Homeyra Sadaghiani $\odot,{ }^{2}$ Anise E. Mansour $\odot,{ }^{2}$ \\ Steven Pollock $\odot{ }^{3}$ and Gina Passante $\odot^{1}$ \\ ${ }^{1}$ Department of Physics, California State University, Fullerton, Fullerton, California 92831, USA \\ ${ }^{2}$ Department of Physics \& Astronomy, California Polytechnic University Pomona, \\ Pomona, California 91768, USA \\ ${ }^{3}$ Department of Physics, University of Colorado, Boulder, Boulder, Colorado 80309, USA
}

(Received 11 August 2020; revised 29 May 2021; accepted 1 October 2021; published 3 December 2021)

\begin{abstract}
As part of ongoing research on student thinking about quantum mechanical concepts and formalism, we explored how students defined and made sense of expectation values. Previous research has focused on student difficulties when defining the expectation value for a generic operator and found that students conflate other quantum mechanical ideas with expectation values. In this study, we analyzed survey data collected from two universities over a number of years and interviews carried out at two points during the semester. With a focus on underlying student thinking, we used a concept image perspective to categorize students' concept definitions of the expectation values in the context of measuring spin in a spin- $1 / 2$ system and in the contexts of measuring energy and position for a particle in an infinite square well potential. Analysis of interview data showed that students invoke many different ideas when explaining their reasoning. The two most common definitions for the expectation value were weighted average and most probable value. In interviews, students' definitions were influenced by whether the problem context involves continuous or discrete observables.
\end{abstract}

DOI: 10.1103/PhysRevPhysEducRes.17.020141

\section{INTRODUCTION}

Quantum mechanics is a notoriously challenging course incorporating complex mathematical formalism and statistical calculations that students must interpret in terms of physics concepts. Quantum mechanical systems are probabilistic in nature. Because of particles existing in superposition states, observable quantities are often not well defined, that is, they do not have a single value that would be measured with $100 \%$ probability. In such situations, it is often useful to use the expectation value, or predicted mean value. The expectation value is the average value that results from measuring many identically prepared particles, an idea which is commonly defined as the ensemble average. Mathematically, the expectation value is equivalent to an average of the possible measurement values weighted by the probabilities, making "weighted average" a suitable definition. In addition to being a useful quantity to calculate for single-particle systems, it is also an important statistical quantity when the system consists of many particles.

Published by the American Physical Society under the terms of the Creative Commons Attribution 4.0 International license. Further distribution of this work must maintain attribution to the author(s) and the published article's title, journal citation, and DOI.
Throughout quantum mechanics, students are asked to solve for and reason about expectation values for both discrete and continuous observables. Depending on the system and how information about the system is represented, there are different mathematical operations that could be employed to calculate the expectation value: matrix multiplication, integration, or summation of values weighted by probabilities. It is certainly possible to carry out any method with little attention to the underlying meaning of the expectation value. However, being able to draw on the meaning of the expectation value is important to determining methods and interpreting the results of a calculation.

Much prior research has focused on identifying student difficulties with many quantum mechanical topics (see Ref. [1] for an overview). Related to the expectation value, overall findings have indicated that students have difficulty distinguishing between the measured value, probability, and expectation value [1,2], and confuse uncertainty, probability density, and expectation value [3]. Recent work has shown that as students are constructing and reasoning about the expressions for expectation values for a generic operator $\hat{Q}$, they rarely connect the calculation to the meaning of the expectation value as the average of many measurements $[1,4,5]$. Additional research exploring student difficulties with time dependence reported that, often, students do not recognize the expectation value of energy is time independent $[1,6,7]$. 
Other researchers have studied students' work with the expectation value to look at use of Dirac notations, matrices, and wave functions [8,9]. Gire and Price note that some students' difficulties with quantum mechanical topics may be due to the notational systems rather than students not understanding the meaning of the expectation value. A separate study used the activation, construction, execution, reflection framework [10] to break down students solving an expectation value problem while calculating the lower bound of $\Delta A \Delta B$ for different operators into phases of activation, construction, execution, and reflection [11]. Researchers reported that most students were not cued toward working with the expectation value and instead tried to solve the side of the equation involving uncertainty.

As part of our previous investigations into students' implementation and preferences of the different methods used to calculate expectation values, we conducted a set of longitudinal interviews in a spins-first quantum mechanics course [12]. We found that for a spin state for a spin- $1 / 2$ particle, students were more confident in a matrix calculation as compared with summing the product of eigenvalues and their probabilities. In both the spin context and the context of continuous wave functions for an infinite square well potential, the main challenge for students when implementing the summation method involved identifying and expressing the quantities within the equation. In some cases, students would identify the correct components of the summation expression but were unsure which values to include.

In this paper, we expand on our preliminary work [13] to explicitly focus on exploring undergraduate quantum mechanics students' underlying conceptual understanding and definitions of the expectation value. We believe this, and the corresponding contextual dependence of their ideas, helps provide useful guidance to future instructional design and deepens our insights into student thinking in this topical area. We explore this conceptual understanding in line with the following research questions:

1. What meaning do students give the expectation value?

2. What type of values do students think the expectation value can have?

3. How does students' thinking of the expectation value depend on the problem context (spin, energy, position)?

We began our investigation with a survey of three quantum mechanics courses to determine to what extent our previous observations were present in these student populations. By asking students to write the meaning of the expectation value, we explore how students define the expectation value in the absence of any problem context. Motivated by the responses students provided, we conducted interviews to investigate the various meanings that students attribute to the expectation value as they are working with different quantum mechanical systems (i.e., a discrete spin system or a continuous system describe by a wave function). We organize these data with a concept image perspective [14], described in Sec. II, with the purpose of categorizing the different ways students define and refer to the expectation value. This focus complements previous literature, which has addressed the expectation value outside of a problem context or focused on difficulties in problem solving, by providing insight into how students think about expectation values as they appear in different quantum systems.

\section{METHODOLOGY}

For the purposes of this study, we collected data in two modalities. First, we administered surveys in three quantum mechanics courses. Based on the results of these surveys, we designed individual student interviews to further probe students understanding. This study builds on previous work that has identified students' use of different methods to solve for the expectation value $[12,13]$. Survey and interview data were collected at two universities. Both universities are large, public, Hispanic-serving, primarily undergraduate institutions. Surveys were administered in both position-first and spins-first quantum mechanics courses. While both courses cover the same topics, the difference is in the order of presentation, with position-first quantum mechanics courses introducing quantum mechanics in the context of position wave functions while spins-first begins in the context of spin$1 / 2$ particles. In this section we describe the design of the survey and interview questions, detail the data collection and student populations, and outline our theoretical perspective for interpreting student responses.

\section{A. Survey}

The primary goal of the written survey was to answer research questions 1 and 2, and to verify our classroom observations that, while students are often able to calculate an the expectation value, many students do not correctly interpret the meaning of this quantity and how it related to the physical system. In particular, we observed that some students correctly interpret the expectation value as an average, while others incorrectly interpret the expectation value as the most likely outcome.

\section{Question design}

The survey questions designed for this study are given in Fig. 1. Question 1 (Q1) is open ended and asks students to explain what the expectation value of a physical observable means to them in words. This question is given without a specific problem context and was asked first, as we did not want to cue students to think in a particular way. The second question was more targeted and directly asked students multiple-choice questions about the types of values that an expectation value can be. For example, question 2a (Q2a) asks whether the expectation value is 
Q1. What does expectation value of a physical observable (momentum, energy, position, spin, etc.) mean to you in words?

Q2. Analyze the following three statements regarding the expectation value for a quantum system. Circle the appropriate word for each and explain. Please explain your reasoning for each - briefly but clearly!

a) The expectation value of an observable is equal to the most probable measurement outcome.

Always Sometimes Never

b) The expectation value of an observable is equal to one of the possible measurement outcomes.

Always Sometimes Never

c) The expectation value of an observable could be... (Circle all that apply)

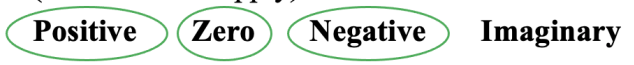

FIG. 1. Survey questions analyzed as part of this project. Correct responses to Q2 are circled.

always, sometimes, or never equal to the most probable value or one of the measurement outcomes. The other two questions ask about whether the expectation value is always (or sometimes or never) one of the possible measurement values, and finally, whether the expectation value can be a negative, zero, or an imaginary number.

\section{Data collection and analysis}

The survey was administered to a total of 90 students over three courses in both position-first (PF) and spin-first (SF) instructional paradigms at two different institutions. The course identified as PF1 was a senior-level course sequence $(N=30)$ at University A using the position-first textbook "Introduction to Quantum Mechanics" by Griffiths [15]. Course SF2 was also a senior-level course at University A $(N=39)$, but taught using the spins-first textbook "Quantum Mechanics" by McIntrye [16]. Course SF3 was a junior-senior-level course at University B $(N=21)$, also taught using the spins-first textbook by McIntyre [16]. All three courses were lecture based and taught by different instructors. While both SF2 and SF3 were lecture based, each included interactive elements of clicker questions and used research-based tutorials [17] in either lecture or recitation sections. In all three courses, versions of these survey questions were administered on paper at the end of the course during lecture. PF1 administered Q1 and an alternate version of Q2; SF2 administered Q1; and SF3 administered both questions 1 and 2 as shown in Fig. 1. All students present in class that day completed the survey (approximately $90 \%$ of enrolled students).

In analyzing these data, we openly coded Q1 for the key element in each student's definition of the expectation value. The data were coded independently by two of us, and each code was then grouped into one of a small number of mutually agreed upon categories. As the goal of this survey is not to quantify different student definitions, we do not report exact percentages of responses within each category, but rather provide qualitative examples of the types of responses student gave. Q2 was primarily used to help provide additional insight into student responses to Q1.

\section{B. Interviews}

In an effort to expand on our findings from the survey and to answer research question 3 , interviews were conducted in the following year in spin-first courses at University A and B. To investigate the impact of problem context on students' definition for the expectation value, interviews were conducted at two points: first, following instruction on the expectation value in the spins portion of the course, and second in the final weeks of the course following instruction on wave functions.

\section{Question design}

The state $\left|\psi_{1}\right\rangle$ in the spins context (see Table I) was given to students in the first set of interviews conducted approximately halfway through the course. Students were asked to calculate the expectation value of spin in the $y$ direction for a state provided in the $S_{y}$ basis. Once students arrived at an answer they were asked "Does that answer make sense to you as an expectation value for this state?" and asked to elaborate. As part of the interview protocol, students were also asked whether there were other methods that could be used to calculate the expectation value. If the answer from the second calculation was different than the first, students

TABLE I. A list of the states or wave functions given to students during the interviews along with the expectation values they were asked to calculate. The answers for $\langle x\rangle$ for both wave functions were given to students. $\phi_{n}$ in $\psi_{2}$ refers to the energy eigenstates for the infinite square well potential. $E_{1}=\pi^{2} \hbar^{2} / 2 m L^{2}$ was given as part of telling the students the results of the expectation values of energy.

\begin{tabular}{lcc}
\hline \hline & State or wave function & Expectation values asked and answers \\
\hline Interview set 1 & $\left|\psi_{1}\right\rangle=\frac{3}{\sqrt{34}}|+\rangle_{y}-\frac{5}{\sqrt{3} 4}|-\rangle_{y}$ & $\left\langle S_{y}\right\rangle=\frac{-8}{17} \frac{\hbar}{2},\left\langle S_{z}\right\rangle=\frac{-60}{68} \frac{\hbar}{2}$ \\
Interview set 2 & $\psi_{2}(x)=\frac{3}{5} \phi_{2}-\frac{4}{5} \phi_{3}$ & $\langle H\rangle=7.2 E_{1}=7.2 \frac{\pi^{2} \hbar^{2}}{2 m L^{2}},\langle x\rangle=0.59 L$ \\
Interview set 2 & $\psi_{3}(x)=\sqrt{\left(\frac{30}{L^{5}}\right) x(L-x)}$ & $\langle H\rangle=1.01 E_{1}=1.01 \frac{\pi^{2} \hbar^{2}}{2 m L^{2}},\langle x\rangle=\frac{L}{2}$ \\
\hline \hline
\end{tabular}


were asked to make sense of the new answer and reconcile their two results. This protocol was repeated for the expectation value of spin in the $z$ direction for the same state $\left|\psi_{1}\right\rangle$.

The second set of interviews were conducted in the last two weeks of the quantum mechanics course and involved two wave functions, $\psi_{2}(x)$ and $\psi_{3}(x)$ for a particle in an infinite square well potential (see Table I). Students were first given $\psi_{2}(x)$, which is explicitly written as a superposition of energy eigenstates. They were asked to find the expectation value of energy and then make sense of their answer. If a student identified summation or matrix methods to calculate the expectation value of energy, they were allowed to carry out the full calculation and were subsequently asked whether the answer made sense as an expectation value for this state. If a student only identified integration as a method, they were only asked to set up the expression and were provided with the result of the calculation and asked to make sense of the value. After answering questions about the expectation value of energy, students were asked about the expectation value of position for the same state. The protocol was repeated with $\psi_{3}(x)$, which was not given in terms of energy eigenstates but instead as a parabolic function of $x$.

Students were not asked to carry out any integration since the focus of the interviews was on the discussion of expectation values and not on their ability to carry out integration. If students began to discuss what the graph of the wave function looked like, they were asked to try to sketch it. Regardless of their success in drawing the graph, they were provided a printed picture of the function following their attempt (see Fig. 2). Students who did not graph spontaneously were given the graphical representation only after they finished making sense of the expectation value calculations on their own. Upon receiving the graph, students were asked whether or not the graph aligned with how they thought of the expectation value so far.

In both sets of interviews, students were asked "What is your definition of the expectation value in the context of [spin, energy, position]?" This question was asked after each time they had reasoned about the results of the calculation in each problem context with the purpose of getting the student to synthesize their interpretation of the

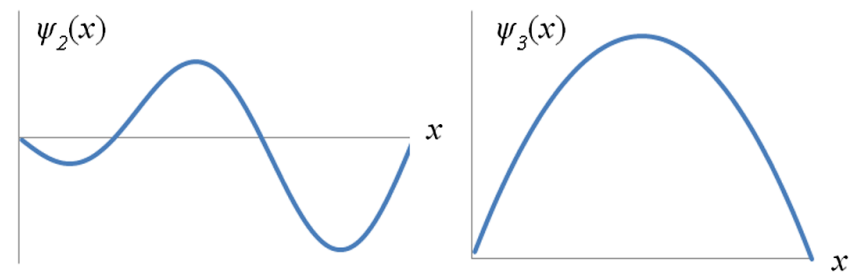

FIG. 2. Graphs that were provided to students for the wave functions $\psi_{2}(x)$ and $\psi_{3}(x)$. expectation value and seek consistency with any formal definition they knew.

\section{Data collection and analysis framework}

Interviews were conducted at both universities (A and B) with student volunteers enrolled in spin-first courses in the year following the survey administration. Interviews involved additional questions not considered in this study and ranged from thirty minutes to no more than an hour. Interviews were videotaped and later transcribed for analysis. We collectively analyzed interview data from both universities. This was done in part due to the limited number of students and because the main focus of this research is on qualitatively identifying students' definitions for the expectation value.

The first set of interviews $(N=20)$ were conducted during the spins portion of the course after instruction on expectation values. The second set of interviews $(N=12)$ were carried out during the last few weeks of the semester after students had learned about expectation values of wave functions in the infinite square well potential. A significant portion of the students interviewed in the second set of interviews (10 of the 12) were also interviewed in the beginning of the semester. The overlap in student participation means that the data capture several individual students' thinking about the expectation value across problem contexts. For the purposes of tracking and presenting student data across multiple interviews, students were assigned pseudonyms, each starting with a different letter of the English alphabet to more easily distinguish them.

For the analysis of the interviews, we explore students' knowledge of the expectation value from a concept image perspective [14]. This perspective originated in undergraduate mathematics education research but has more recently been used in physics education research to categorize student thinking about vector calculus concepts in electrodynamics [18-21]. A concept image is a multifaceted and dynamic construct. It encapsulates students' thinking about and ideas associated with a given topic. A concept image can include equations, graphs, pictures, definitions, and specific phrases. Taking the expectation value as an example, a concept image could include (but is not limited to) histograms, different methods of calculations, and the definition of the expectation value as an average. Depending on the particular problem context, some subset of the concept image is evoked for the purpose of problem solving, yielding an evoked concept image. A concept image may even contain contradictory elements that only create conflict when they are evoked together. Likewise, a student's concept image may only contain fragmented ideas that the student may relate to a topic but struggle to express coherently.

The way in which a student specifies the meaning of a topic is referred to as the concept definition. A concept 
definition is something personal to a student and may differ from the formal (textbook) definition of a topic (for example, a commonly accepted formal definition for an expectation value is an ensemble average). It is the meaning a student attributes to a topic and thus can be part of a student's evoked concept image. Specifically, Tall and Vinner describe the concept definition as the explanation for the evoked concept image [14]. However, it is possible that the concept definition may be disconnected from the way the student solves a given problem. A student may describe an expectation value as being weighted by probabilities but not connect this to the summation method. In this case, the definition and summation method could be seen as disconnected elements of an evoked concept image.

A concept definition can be reconstructed at any time, just as a concept image can change over time as a student accrues and incorporates new information. Given the dynamic nature of the concept image, Tall and Vinner note that students may develop a restricted concept image based on an overuse of a formulaic approach, even if the formal definition is taught. In this case, a concept image is dominated by a limited set of ideas. As an example, a student could be taught the definition of the expectation value along with both the summation and matrix methods and subsequently go on to spend weeks calculating expectation values without activating the definition as part of their cognitive structure. Then in a broader problem context or in an analogous situation, such as defining the expectation value or deriving the summation method for a superposition of energy eigenstates, they may draw on different connections rather than on the generalized meaning associated with the expectation value. Tall and Vinner further add that students may "even have been taught to respond with the correct concept definition whilst having an inappropriate concept image" [14]. Considering expectation values, this could manifest as a student defining the expectation value as a weighted average but not connecting the definition to the physical system or mathematical expression.

In the present study, interviews were conducted to probe more deeply into students' ideas of the expectation value that were identified in the surveys. For the interviews, we applied a concept definitions lens and allowed defined categories to be emergent from the data. This analytical lens is aligned with that of phenomenography [22]. Phenomenography is a qualitative approach that aims to identify and categorize the variations among individuals' perception and conceptualization of phenomena. The resulting categories are based on students' descriptions. A set of categories appearing in multiple situations is considered stable for that student [22]. Previous research in electricity and magnetism has used phenomenography to categorize student ideas related to vector operators [20] and magnetic fields [23].

Both video and transcripts were used to identify specific concept definitions reflective of ideas expressed by the students. Analysis specifically focused on responses to two questions: "How do you make sense of your result as an expectation value?" and "What is your definition of the expectation value of [spin, energy, position]?" Student responses to both questions were collectively coded based on key phrases. The specific phrases were then labeled with a particular concept definition.

To be labeled with a specific concept definition, say weighted average, a student would need to explicitly state that the expectation value was either a weighted average or "closer to the higher probability" since the idea of a weighted average definition is an average weighted by the probabilities. Concept definitions were defined in our analysis by directly drawing from student phrases. In many cases, different students used the same phrasing when discussing their definition of the expectation value or the result of their calculation. This meant there was some prevalence to that concept definition across students. In specific problem contexts, individual students conveyed definitions that were not shared with other students. These definitions were also labeled as concept definitions based directly on the students' responses. We report both the common and unique ideas with the purpose of capturing as broad a picture of student thinking about the expectation value as possible.

The resulting list of concept definitions were iterated over for consistency and subject to discussions within the research team about what the concept definition meant or included. We do not claim to have produced a complete list of concept definitions for every possible problem context. However, we expect that many responses within new contexts would fit within our categories.

\section{SURVEY RESULTS}

The written surveys asked students to explain what the expectation value means to them using words and explicitly prompted them to not use equations. Two authors independently coded student responses. These codes were then placed into one of four main categories: average, most likely, probability related, and other. In all three classes (PF1, SF2, and SF3), student responses fitting in each of the first three categories were present with at least $5 \%$ of responses fitting into each. However, while it was possible to group student responses into these four categories, there was significant variation in the types of responses.

\section{A. What meaning do students give the expectation value?}

In this section we focus on student responses to Q1, Q2a, and Q2b. We provide some examples of student responses to Q1 and connect them with their answers to Q2 whenever possible. (The text of these questions is provided in Fig. 1.) A small number of responses were not captured by the three main categories described below and were coded as "other." Repeated responses in this catch-all category provided 
equations only, indicated the expectation value is the measurement outcome(s), or left the question blank.

\section{Expectation value is the... average}

Within the average category, we found a small number of responses that provided close to what we would consider a textbook definition. For example,

The average value of that physical observable when it is measured in many similarly prepared systems. (PF1)

However, this student did not go on to answer all subsequent questions correctly. In response to Q2a, this student correctly identified that the expectation value will only sometimes be the most likely value, but they then indicated that the expectation value will always be one of the possible measurement outcomes $(\mathrm{Q} 2 \mathrm{~b})$. In their explanation for Q2b, they drew on the example of a spin-1/2 particle where they correctly provided the range for an expectation value $(-\hbar / 2<\langle S\rangle<\hbar / 2)$, but then wrote "it has to be at least $-\hbar / 2$ (which is one of the outcomes of a spin-1/2 measurement). We cannot expect anything smaller than $-\hbar / 2$." This student's explanation seems to be in contrast to their selected answer.

Another student response that fit into the average category did not reference ensemble measurements, but did specify that the average was weighted by the probabilities:

It refers to the overall weighted average of all the possible physical observable values, which isn't necessarily possible to be observed itself. (SF3)

This student went on to correctly answer all parts of Q2, using sketches to explain their answers (see Fig. 3).

There were other students whose responses to Q1 were much less informative and they simply said something like "It is the mean (SF3)," or "the expectation value of an observable is analogous to finding the "average" value in classical terms (SF2)."

Question 2a explanation for

"the expectation value of an observable is sometimes the most likely value":

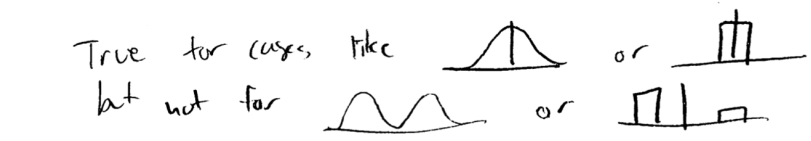

Question 2b explanation for

"the expectation value of an observable is sometimes one of the measurement outcomes": i.e.)

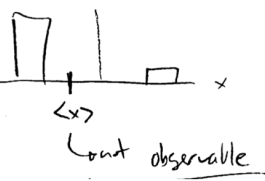

i.e.)

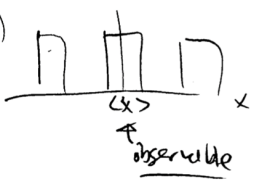

FIG. 3. Student explanations for their responses on questions 2a and 2 b. Student is from class SF3.
Finally, there were some responses that fell into this category, but after reviewing their explanations, it was unclear whether the student really understood the meaning of the definition they were providing. The following student explanation illustrates this:

The expectation value of a physical observable is the average of the values expected, the weighted average of the eigenvalues (what we can expect to measure). (SF2)

In this example, the first part of the response is correct, but then it ends with the parenthetical "what we can expect to measure." This gives the impression that the student might think the expectation value is both the average and the most likely.

In almost all instances, students whose responses fell into this average category were consistent in their response to Q2a and indicated that the expectation value is only sometimes equal to the most likely outcome.

\section{Expectation value is... most likely}

Other students explained that the expectation value tells us the most likely measurement outcome. Examples of student responses are

The expectation value is the value that the observable would most likely have according to the wave function. (PF1)

The most expected value of the physical observable would occur. (PF1)

I think of the expectation value as giving the "most likely" (or most average) value of whatever your measuring based on the probabilities of observing that state; i.e., particle in infinite potential well of length $L$,

$\langle x\rangle=L / 2$ exactly half way or most likely position. (SF2)

Some responses were a little more nuanced. For example, consider the following student response:

The expectation value means the region where the particle is likely to be measured. (SF3)

It clearly states that the expectation value tells us the most likely measurement, but instead of referring to the expectation value as providing a single value, the student indicates that the expectation value indicates the region where the particle is likely to be found. There were several responses that referred to the expectation value as providing a range of values in both this category, as well as in the probability-related and other categories.

In other instances, the student provided correct (or partially correct) information about how to calculate the expectation value, but then referred to it as the most likely value: 
The expectation value is the sum of all probabilities and so gives the position or state most likely to be found, depending on what you are observing. (SF2)

As with the responses in the average category, students were very consistent between their definition and their answer to Q2a, where almost all of them indicated that the expectation value is always the most likely value.

\section{Expectation value is... probability related}

The responses in this category are closely related to those in the most likely category, however they often speak more to the probabilities that different values can take rather than focus on the single most likely result.

It means the probability of a physical observable occurring. (SF3)

It means how often will I find this particle in some state. (SF2)

It means the probability of a physical observable occurring. (PF1)

As with the previous category, there were instances where students indicated that they knew how to calculate the quantity, but assigned an incorrect meaning to it:

How probable to get each option. $\sum($ value $) *($ prob $)$ (SF3)

Most of the responses in this category connect the expectation value to a range of values as opposed to a single number. Unlike the other two categories where student responses were very correlated with their answer to Q2a, students in this category more commonly indicated that the expectation value is always the most likely value (in response to Q2b), but there were several instances where they correctly indicated that this was not always the case. As we later found in the interview results, these two categories of most likely and probability related are not necessarily distinct. In the interview analysis we combine these two categories in an effort to explore how students relate the expectation value and probability.

\section{B. What type of values do students think the expectation value can have?}

In the previous section, we identified some of the common responses when we asked students what the expectation value meant to them. In Q2, we more directly asked whether or not students agreed with some common statements we have heard in our collective experience teaching quantum mechanics.

In response to Q2a, the most common answer was that the expectation value is sometimes equal to the most likely answer, provided by 29 of the 51 students in the combined PF1 and SF3 datasets. A further 18 (of 51 students) indicated that it was always the most likely answer.
Results were similar to Q2b with $31 / 51$ correctly selecting "sometimes" and only 12 selecting "always" as their answers. As mentioned previously, these answers correlated with their definitions to Q1 when their definitions fit into the average and most likely categories.

Results to the final question (Q2c) were mostly correct, with a handful of student indicating that either the expectation value had to be a positive number or that it could be imaginary. The explanations to this question rarely provided additional insights as these students often provided incorrect declarative statements without any additional explanation. Sample student explanations are provided below.

\begin{abstract}
The expectation value can be a positive real number but it can also have an imaginary part just like the expectation value of momentum. (PF1)

Prob is always $\geq 0$, but value can be real, imaginary, positive, or negative. (SF3)
\end{abstract}

\section{INTERVIEW RESULTS}

Analysis of student interviews allowed us to explore how student understanding of the expectation value changed across the differing problem contexts of spin, energy, and position over the semester. In all problem contexts, the expectation value is formally defined as the average of the measurements made on many particles with identical states or as a weighted average. In this section we outline students' ways of thinking about expectation values as they make sense of answers to expectation value problems and define the meaning of the expectation value.

Analysis of the survey data yielded several categories related to the meaning students attributed to the expectation value, with two being the most common: the expectation value as the "average value" and the expectation value as the "most probable value." In analysis of the interview data, we parse both of these concept definitions into separate categories depending on how the students articulated their definition of the expectation value. Variations on the average value include "ensemble average," where students acknowledge that there are many measurements being taken and can discuss probability of measurements and weighted average, where students focus on the probabilities for a state. It is possible for students' responses to be labeled with both ensemble average and weight average concept definitions when they connect an expectation value to both probabilities and many measurements.

Additionally, there were several other evoked concept definitions identified that appeared in the associations students made during the problem-solving interviews (e.g., the expectation value as the probability of measuring a value). These definitions, as well as their dependence on the problem context, allow us to further explore the probabilityrelated category. Some definitions only appeared in specific problem contexts, while not in others. We identify the range of definitions to shed light on how students make sense of 
TABLE II. Concept definitions identified in the responses of all students that were interviewed. An entry of " 0 " implies that the concept definition did not appear in that context for any of the interviewed students. Some students were labeled with multiple concept definitions.

\begin{tabular}{|c|c|c|c|c|c|}
\hline Category & Concept definition & Meaning & $\langle S\rangle N=20$ & $\langle H\rangle N=12$ & $\langle x\rangle N=12$ \\
\hline \multirow[t]{4}{*}{ Average } & Ensemble average & Average over many measurements of the same state & 2 & 1 & 1 \\
\hline & Weighted average & Average is weighted by probabilities & 13 & 6 & 3 \\
\hline & Average of values & Unweighted average of the eigenvalues & 1 & 0 & 0 \\
\hline & Ambiguous average & Statement of average without further explanation & 0 & 2 & 0 \\
\hline \multirow[t]{3}{*}{ Probability } & Probability of value & Probability of an eigenvalue & 3 & 2 & 1 \\
\hline & Value you expect & Answer tells you what value is more likely & 2 & 1 & 1 \\
\hline & Most probable value & Eigenvalue with the highest probability & 0 & 0 & 4 \\
\hline \multirow[t]{4}{*}{ Other } & Summation & The result of mathematical calculation & 0 & 2 & 0 \\
\hline & $\begin{array}{l}\text { Difference in energy } \\
\text { levels }\end{array}$ & $\begin{array}{l}\text { Difference between energy levels in an infinite } \\
\text { square well potential }\end{array}$ & 0 & 1 & 1 \\
\hline & Related to wavelength & $\begin{array}{l}\text { Answer tells something about the wavelength } \\
\text { of wave function }\end{array}$ & 0 & 0 & 2 \\
\hline & Equal area & $\begin{array}{l}\text { Position where area under the wave function } \\
\text { is equal on both sides }\end{array}$ & 0 & 0 & 1 \\
\hline
\end{tabular}

expectation values in relation to specific quantum mechanical observables. Table II provides a list of the different concept definitions and identifies the number of students labeled with each concept definition in the three expectation value contexts: spin $(\langle S\rangle)$, energy $(\langle H\rangle)$, and position $(\langle x\rangle)$.

The following sections present students' concept definitions in each of the problem contexts and then subsequently discusses how these definitions changed for the ten students who were interviewed at both points in the semester. In the discussion of the interview results, we do not give a detailed description of the methods that students used for calculating EV as those results are part of another study [12].

\section{A. Making sense of spin expectation values}

In the spins interview, students worked with the state, $\left|\psi_{1}\right\rangle=\frac{3}{\sqrt{34}}|+\rangle_{y}-\frac{5}{\sqrt{34}}|-\rangle_{y}$, and were asked to calculate expectation values for the $S_{y}$ and $S_{z}$ operators. The $\langle S\rangle$ column of Table II enumerates the different evoked concept definitions related to the expectation value of spin operators.

When asked to define and make sense of their expectation value calculations, 2 of the 20 students' concept definitions included many measurements, as exemplified by the following.

Syd: If we ran it [the experiment] for how many ever times, ... it's the average value of all the results

Syd's concept definition for the expectation value in a spin$1 / 2$ system aligns with the ensemble interpretation that is part of the formal expectation value definition. Both of the two student responses discussing many measurements were labeled with an ensemble average concept definition.
Most commonly, students' evoked concept image included elements associated with a weighted average perspective $(13 / 20)$. However, only six of the 13 students explicitly defined the expectation value as a weighted average, including both students with the ensemble average concept definition. The remaining students simply defined the expectation value as the average (7/13) and made sense of their answer by discussing the differences in the probabilities. They further included that the expectation value would be closer to the eigenvalue with the higher probability, as shown below.

Raife: I have a negative value. So I'm thinking this [probability of - $\hbar / 2$ ] would be bigger. The distribution should be more on the left...When we were doing expectation value we did graphs [histograms], so the expectation value is... the average value. So if you're, I guess in my case, we're leaning more toward $-\hbar / 2$.

Raife acknowledges the probability of measuring $-\hbar / 2$ is higher and the expectation value is closer to the value with the higher probability (Fig. 4). The other six students shared similar ideas, explicitly discussing the probability being "weighted more to the left." Most of these students specifically connected their calculation to a histogram representation that is used frequently in their textbook. Because all 13 students explicitly related the expectation value to relative probabilities in this way, their responses were labeled with a weighted average concept definition for the purposes of categorization.

Of the students whose responses were labeled with a weighted average concept definition, roughly half $(6 / 13)$ either did not invoke the associated summation method or identified probabilities or values that were incorrect for a 


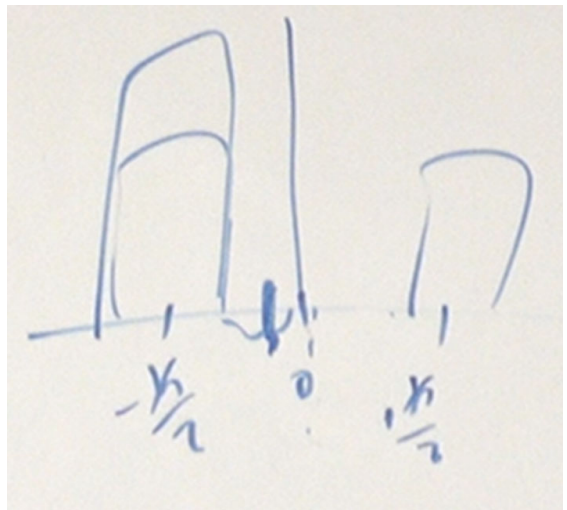

FIG. 4. Raife's depiction of a histogram for spin. He identifies that an equal probability will yield an expectation value of 0 and a higher probability of $-\hbar / 2$ will mean the expectation value will be shifted to the left (thick vertical dash).

given calculation. For example, Harper explicitly said that the expectation value was a weighted average and described the results accordingly. However, as part of their calculation, Harper summed the probability amplitudes and divided by two instead of weighting the values with the probabilities. Three students not using the summation method recognized that their matrix calculation was incorrect after arriving at an expectation value of 0 , because 0 meant the probabilities should be equally weighted, a notion which was in conflict with the probability amplitudes given in the problem statement. The absence or misapplication of the summation method implies a disconnect between students' concept definition and the evoked concept images related to procedure. They said the definition, but did not invoke the equivalent mathematical formalism of an average weighted by probabilities.

The description of the expectation value as the most probable value, as shown in the survey data, did not occur in any interviews in the spins context. However, ideas of probability were included in students' concept definitions in other ways. A few students $(3 / 20)$ described the result of their expectation value calculation as the probability of getting a value. After calculating the expectation value for spin in the $z$ direction using matrices, Freya arrived at the answer of $-\frac{60}{68} \frac{\hbar}{2}$. When asked to discuss the meaning of the value, she stated the following:

Freya: If you were to get an experiment in this state and measure it in the $S_{z}$ you would have a probability of getting $-\hbar / 2$ of $\frac{60}{68}$

We identify this as a "probability of value" concept definition. This concept definition appeared in two other interviews where students would take the fraction before the eigenvalue and claim that was the probability of measuring the eigenvalue. This is similar to how the coefficient before a state in a superposition is related to the measurement probability associated with a given eigenvalue. Freya was the only one of the three who checked this line of reasoning by calculating the actual probability of $-\hbar / 2$. When the probability did not match her expectation value, she stated the probability description was incorrect but that she could not come up with an alternative definition.

In line with the survey data, two students, Keith and Leanne, described the expectation value in terms of "what you would expect to measure out of the system" and the "value you would expect." Notably, Keith acknowledges that the expectation value is "related to probability, not just probability." For both students, this way of defining the expectation value came from an attempt at deriving meaning and wasn't just a statement of saying the most probable value. Leanne articulated a little beyond this definition to ease some of her initial discomfort with getting a negative expectation value in her calculation.

Leanne: Hmm, like it sounds like an overly simplistic description but "did you get what we would expect to get out of the measurement." Like I don't know how else to phrase it. It's just like what we would expect to measure out of the system

Interviewer: So how do you interpret that definition in terms of that result [the calculated answer for $\left.\left\langle S_{y}\right\rangle\right]$ ?

Leanne: Well once I put the $-\hbar$ on the outside. That is helping me a little bit because $-\hbar / 2$ is a totally accepted value, like that's not something unusual. It's just, I don't know. It is still strange to me in a sense.

Leanne recognized that $-\hbar / 2$ is a measurement value, but did not connect this to her definition that the expectation value is "what we would expect to measure." As both Keith and Leanne emphasized the idea, we label them with a value you would expect concept definition. Neither student expanded on their response, instead they expressed uncertainty in the conceptual meaning.

\section{B. Making sense of energy expectation values for wave functions}

At the end of the spins-first course, 12 students (10 students identical to those in the spins context) were interviewed. Students were first asked about methods for calculating energy and position expectation values for two wave functions, $\psi_{2}(x)=\frac{3}{5} \phi_{2}-\frac{4}{5} \phi_{3}$, where $\phi_{n}$ is the $n$th energy eigenstate, and $\psi_{3}(x)=\sqrt{30 / L^{5}} x(L-x)$, for an infinite square well. Unlike in the spins context, students were not asked to calculate anything unless they indicated the possibility of using a summation method or matrix method. In this section we focus on students' responses as they make sense of provided answers for the energy expectation values of these two functions.

Individual students' definitions did not change between the two given wave functions. However, throughout the 


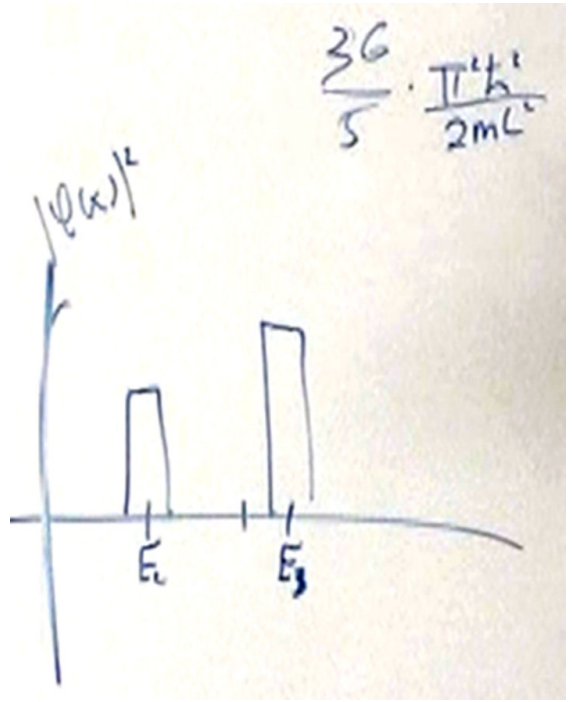

FIG. 5. A histogram Wayne drew to describe the expectation value of energy. He labeled the vertical axis using a square of a function. On the horizontal axis he labeled $E_{2}$ and $E_{3}$ and plotted the probabilities. He then drew a tick mark closer to $E_{3}$ because there is a higher probability of measuring $E_{3}$.

interview, two students indicated multiple definitions or ways of thinking about the expectation value, and were thus counted as exhibiting more than one evoked concept image. The $\langle H\rangle$ column of Table II enumerates the different concept definitions for expectation values of energy.

Just one student defined the expectation value as the "average if you took a bunch of measurements" of energy in line with the ensemble average concept definition. This student also had a response consistent with a weighted average concept definition along with five others. The weighted average concept definition was the most common means of discussing the expectation value. Consistent with responses to spin questions given earlier in the semester, all 6 students defined the expectation value as an average value, but made sense of their results by discussing the value as closer to the higher probability (5), as Mark does below.

Mark: We're much more likely to get energy $3\left[E_{3}\right]$ because the prob of getting it is higher so it makes sense...this [7.2] is way closer to the 9 then the 4.

Mark connects the answer 7.2E 1 to the probabilities of the different possible energy eigenvalues which he also wrote in terms of $E_{1}: E_{2}=4 E_{1}$ and $E_{3}=9 E_{1}$. We identify Mark and other similar responses with the weighted average concept definition because of the explicit emphasis on the answer being closer to the most probable value. For some students the interview was the first instance they made a concrete connection between the calculation and the definition as a weighted average. For example, after recognizing $7.2 E_{1}$ fell between $E_{2}$ and $E_{3}$ and was closer to the more probable value, Gil acknowledged that he had previously "never thought about it that way."

Two students described the expectation value solely as an average. They drew on the meaning of average to acknowledge that the expectation value was within an appropriate range, "somewhere between states 1, 2, and 3." Since these students did not include the same weighted average language of "closer to" or "more toward" that is associated with attention to the probabilities of the values, even after further probing from the interviewer, they are just labeled as having an "ambiguous average" concept definition. This does not mean that the students would not invoke weighted average ideas within another problem context, but that in response to our interview questions, they did not evoke these ideas.

Wayne, who used weighted average ideas when making sense of $\langle H\rangle=1.01 E_{1}$ for $\psi_{3}$, was also the only student to reference the energy expectation value as the "value you would expect."

Wayne: Um, well it is the value that we would expect the energy to be at. Its like the average of what the energy could be... so since this is so close to 1, that means that $E_{1}$ is very heavily weighted and whatever other energy states are involved are much, much smaller.

Wayne's explanation when working with $\langle H\rangle$ includes contradictory ideas. Expanding on the value we would expect, which has the connotation of being the most probable value, Wayne articulates the expectation value as an average that is closer to the more heavily weighted value. However, based on his definition, we identify him as having both the weighted average and value you expect concept definitions since he included both when making sense of $\langle H\rangle$ for $\psi_{2}(x)$ and $\psi_{3}(x)$.

Two students' responses were labeled with the probability of value concept definition. Just as in the spins context, the value or fraction preceding the eigenvalue was viewed as the probability for measuring the associated eigenvalue. This was contradictory for both students because the given answer was $7.2 E_{1}$ and the state was given as a superposition of $E_{2}$ and $E_{3}$ eigenstates. Keith went on to try to synthesize a definition for the expectation value but did not arrive at one.

Keith: I always think it's what you'd expect. It's kind of like a probability of getting a certain value. Well, I also know it is not necessarily a probability. We're dealing with probability amplitude, but you're not squaring it. So it's almost like a measurement but it's not necessarily a measurement.

Keith's evoked concept image when making sense of $\langle H\rangle$ included several different quantum mechanical quantities relevant to the expectation value. While attempting to articulate a coherent definition Keith introduced and 
dismissed connections to probability and measured value but did not expand upon "it's what you'd expect." Both the statements of Wayne and Keith highlight a struggle for these students to arrive at a concrete definition of the expectation value even at the end of a quantum mechanics course. Much like the students in the spin context, it is possible that either student was attempting to articulate that the expectation value reveals which of the possible measurement outcomes is the most probable value. Notably, all of these students either set up a correct integral or successfully carried out the summation method. Thus they are often able to compute, but not explain, what the computation means.

Two students, Duncan and Leanne, referenced uncertainty in regards to how to make sense of the responses but defined the expectation value as the "summation of the probabilities times the values," evoking a very procedural response but did not make sense of the actual value beyond the result of mathematical calculation. Duncan, in addition to expressing "summation" and "ambiguous average" concept definitions, also said that the expectation value of energy "just reveals the gap between each of the energy planes [levels]," stating that the distance between $E_{2}$ and $E_{3}$ on an energy level diagram was given by the expectation value.

\section{Making sense of position expectation values for wave functions}

In the same interview where students were asked about the expectation value of energy, they were also asked to make sense of the expectation value of position for both $\psi_{2}(x)$ and $\psi_{3}(x)$. For some students, the concept definition changed when transitioning from an expectation value of energy to an expectation value of position. Three students were identified with multiple concept definitions for an expectation value of position, indicating their evoked concept images included different ideas of how to make sense of the given values. The $\langle x\rangle$ column of Table II summarizes the different concept definitions within this problem context.

The ensemble average and weighted average concept definitions were less common than in the previous discrete contexts. One student, Tristan, indicated both that the expectation value was the average of many measurements and that it is closer to the higher probability, as shown below.

Tristan: ....more area under the curve on this side, ... so then when you average all the measurements out that you made, you're going to find the particle on this side more often when you do a measurement. So average all of the measurements out, the position [the expectation value] is going to be placed more on the side of where the probability is.
Tristan was labeled with an ensemble average concept definition as he made sense of the expectation value in terms of many measurements and the weighted average concept definition to account for his discussion of probability.

Two other students defined the expectation value in terms of an average, but did not invoke the idea of many measurements. One of these students, Wayne, said average and the value you would expect, just as he had in the energy context. For $\langle x\rangle$, however, he arrived at the idea that it was the most likely position, as shown in the pair of excerpts about $\psi_{2}(x)$ below.

Interviewer: So if you do this out, you get 0.59L. How do you make sense of that?

Wayne: It means like the average, like. So I don't know. It should have some curve, but like, it will probably be largest around [draws a single peak over the location of the expectation value]. Maybe not even largest, but it is going to have like a distribution where this will be the most heavily weighted spot. but it might not be that. it might be weird.

Following this discussion, Wayne was provided the graph for $\psi_{2}(x)$ (Fig. 2). He acknowledged that is was a combination of the two energy eigenstates, $\phi_{2}$ and $\phi_{3}$, for the infinite square-well potential and connected the negative coefficient associated with $\phi_{3}$ to the shape of the graph. A cursory analysis of the graph reveals that $0.59 \mathrm{~L}$ is not near a local maximum or minimum. However, when asked for his definition for the expectation value of position, Wayne continued to discuss the expectation value in terms of the most likely value. This reasoning is consistent with his drawing of a single peak over the location of the expectation value when asked to make sense of the result.

Interviewer: So I asked you about the conceptual meaning of the expectation value when we were working with energy. What is the conceptual meaning of it [the expectation value] in this [position] context? How are you thinking about it?

Wayne: It is the value we would expect $x$ to be if we measured it. It is the most likely, just because of the way the probability density is weighted.

Interviewer: So it is the most likely value?

Wayne: Yeah, but that does not mean that it would be measured. It is the average.

Transitioning to the position context, Wayne's concept definition includes most probable value as a direct extension of the value we would expect. Even though Wayne also includes aspects associated with a weighted average definition, when asked for clarification he reaffirms that it is 
both the most likely value and the average. As such, Wayne's response is identified as having three concept definitions.

The most probable value concept definition was the more commonly evoked for the expectation value of position. In total, four students, including Wayne, made a connection between the expectation value and the most likely or most probable value. In the following example, Carlos directly connected the expectation value to the most probable value.

Carlos: The expectation value is the place with the highest probability of finding the particle.

This kind of response in the interviews is consistent with the most probable value definition for the expectation value on the survey given at the end of the semester without any contextual frame. Since the most probable value concept definition did not occur in the spins or energy expectation value, findings suggest that responses of most probable on the survey were thinking about $\langle x\rangle$ and continuous wave functions.

Other concept definitions were only identified in responses made by individual students. These included "related to wavelength," "difference between energy levels," "probability of finding $x$," and "equal area." The related to wavelength concept definition appeared as one student connected the graph of $\psi_{2}$ to its expectation value $\langle x\rangle=0.59 \mathrm{~L}$. One student equated the expectation value with probability, stating the result of calculation was "the probability of finding $x$ at a certain point within the well," which we count toward the probability of value concept definition and specifically connected the result of calculation to the area under the curve. This conflation of probability and the expectation value is consistent with findings in previous literature [1,2]. Duncan defined the expectation value of position as the difference between energy levels, identical to his use of this definition for the energy expectation value. One student said that the expectation value is the location where the area under each side of the graph is the same. We label this as the "equal area" concept definition. This is similar to students' belief in classical mechanics that "center of mass" divides an object into two pieces of equal mass [24].

In a few cases, comparing the graphs of the two functions (Fig. 2) led students to conclude their definition of most probable value or related to wavelength did not make sense and they decided they were unsure of what the expectation value meant. Leanne articulated a struggle to understand the conceptual meaning of the expectation value, after she abandoned an attempt to connect the wave function to a bar graph representation.

Leanne: I can get answers to problems, but then when I start to try to explain what it actually means I get a little bit like lost...
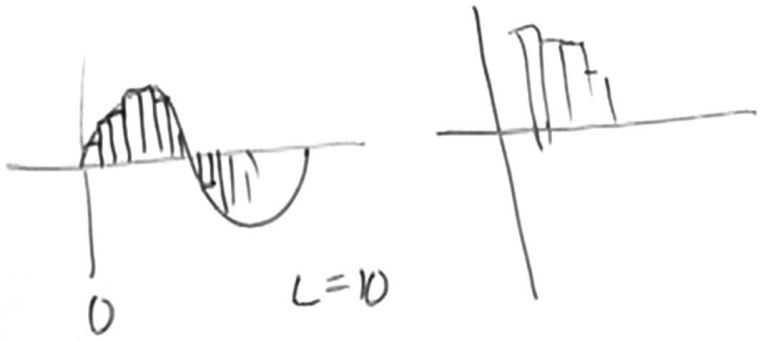

FIG. 6. Leanne's attempt to make sense of the wave function as a histogram. She draws bars for different points of the wave function, but is not able to connect this to the expectation value of position.

Leanne and one other student attempted to draw a histogram for the wave function, but both students expressed they were unsure when asked to connect it to the meaning of the expectation value (Fig. 6).

\section{Comparison of concept definitions across contexts}

Interviews conducted at two points during a spins-first course revealed a number of ideas and definitions that students connected to the expectation value that are similar across problem contexts. Ten students participated in both sets of interviews and answered questions about expectation values of spin, energy, and position. While some students' concept definition remained consistent across different problem contexts, other students' concept definitions changed as the questions switched from the expectation value of spin to $\langle H\rangle$ for an infinite square well potential to $\langle x\rangle$ for an infinite square well potential. The different concept definitions identified in responses for the ten common students are shown in Table III.

Overall, responses for the expectation value of energy were similar to the responses given in the spin- $1 / 2$ context. For the first state, $\psi_{2}(x)$, most students read out the probability amplitudes from the superposition representation and used weighted average reasoning in a similar manner to when working with the spin- $1 / 2$ system. The probability of value and value you would expect concept definitions also reappeared in the energy context when students made sense of their answers for the superposition state. These ideas persisted in students' reasoning about the expectation value of energy for $\psi_{3}(x)$, which was asked later in the interview. Five student responses were labeled with ensemble average or weighted average concept definitions for both the energy and spin expectation values. The sixth student, Duncan (D), who expressed a weighted average concept definition in the spin context, defined the energy expectation value as the difference between energy levels in an infinite square well potential. For $\langle H\rangle$, Gil (G) had initially described $\left\langle S_{y}\right\rangle$ as the probability of getting the value and only invoked weighted average ideas in the second interview later in the semester. 
TABLE III. Concept definitions identified in the responses of the 10 students that were interviewed in both the spin and wave function contexts. Students are identified by the first letter of their pseudonym. A blank entry implies that the concept definition did not appear for any of the interviewed students. A "..." implies the concept definition was present for some students, but not for the 10 students analyzed in this section. Note that some student responses were found to have multiple concept definitions within a single question.

\begin{tabular}{llccr}
\hline \hline Category & \multicolumn{1}{c}{ Concept definition } & $\left\langle S_{y}\right\rangle$ and $\left\langle S_{z}\right\rangle$ for $\left|\psi_{1}\right\rangle$ & $\langle H\rangle$ for $\psi_{2}(x)$ and $\psi_{3}(x)$ & $\langle x\rangle$ for $\psi_{2}(x)$ and $\psi_{3}(x)$ \\
\hline Average & Ensemble average & $\ldots$ & $\mathrm{T}$ & $\mathrm{M}, \mathrm{T}, \mathrm{W}$ \\
& Weighted average & $\mathrm{D}, \mathrm{M}, \mathrm{P}, \mathrm{T}, \mathrm{V}, \mathrm{W}$ & $\mathrm{G}, \mathrm{M}, \mathrm{P}, \mathrm{T}, \mathrm{V}, \mathrm{W}$ & $\mathrm{L}$ \\
& Ambiguous average & $\ldots$ & $\mathrm{F}, \mathrm{K}$ & $\mathrm{W}$ \\
Probability & Probability of value & $\mathrm{F}, \mathrm{G}$ & $\mathrm{W}$ & $\mathrm{F}, \mathrm{G}, \mathrm{W}$ \\
& Value you expect & $\mathrm{K}, \mathrm{L}$ & $\mathrm{D}$ \\
Most probable value & & $\mathrm{D}$ & $\mathrm{D}, \mathrm{P}$ \\
& Difference in energy levels & & $\mathrm{V}$ \\
& Related to wavelength & & \\
\hline \hline
\end{tabular}

Consistently across all three problem contexts, 3 of the 10 students were identified with ensemble average or weighted average concept definitions. Two of these students were consistent in using only these ideas, while Wayne also introduced the conflicting idea of most probable value for the expectation value of position. Victoria, who expressed an ensemble average perspective for $\langle H\rangle$ transitioned to thinking about area under the curve of the wave function. Conversely, Tristan invoked an ensemble average perspective cued by where a particle would be more likely to be found during "all the measurements."

None of the 10 students described the expectation value as the most probable or most likely value when working with spin or energy, but several did when considering the expectation value of position. Three students switched from only a weighted average concept definition to including a most probable value concept definition when moving from the energy to position contexts in the same interview. Two of these three students did not discuss average at all when talking about the expectation value of position. This suggests that the connection between the expectation value and the most probable value, as seen in the survey data, could be an artifact of the wave functions context.

Lastly, there was a greater variety of concept definitions in responses for the expectation value of position as students tried to make sense of the given results. This led to 3 students stating they were unsure of how to define the expectation value of position, whereas they had previously provided a response for the expectation value of spin and energy. For example, Gil initially claimed the expectation value of position was the most probable value but said he was unsure after recognizing that response was inconsistent with the graphs of $\psi_{2}(x)$ and $\psi_{3}(x)$. Notably, this came after Gil used weighted average ideas to describe the expectation value of energy for the same wave functions. The shifts in student responses to most probable value reasoning and the increase in students' uncertainty, suggests that reasoning about quantum properties for continuous observables is an area that can be better supported in instruction or curricula.

\section{CONCLUSIONS AND DISCUSSION}

As part of this research we have investigated how students defined the concept of expectation values without a problem context and across several common problem contexts. Surveys about what meaning students give to the expectation value without the immediate influence of a problem context and the types of values that students associate with expectation values. Interviews extended this analysis to categorize students' personal concept definitions they used to make sense of and define expectation values in the contexts of spins, energy, and position over the course of a quantum mechanics class.

The focus of this work on students' concept definitions is motivated in part by our previous work on student problem solving methodologies [12], but here we concentrate on students' underlying conceptual framing associated with expectation values. Despite being taught the formal definition in class, students incorporate a wide range of ideas when working with expectation values. Furthermore, the incorporated ideas vary as the physical context evolves throughout the semester. While some definitions in the other category were context dependent, the definitions associated with average and probability were common in all problem contexts. The identified concept definitions provide a foundation for both the further exploration of student problem solving in this arena and for the development of targeted instruction and curriculum design.

Surveys found the persistent student idea that the expectation value always returns a possible measurement outcome, which is consistent with interviews where students defined the expectation value as the most probable value or would describe the expectation value as a measurement probability itself. In response to the survey, it was common in all classes for students to describe the expectation value either in terms of an average or as the most likely value. Notably, no student referred to the expectation value as the most probable value during interviews dealing with spin or energy expectation values. However, probability-related definitions did appear. 
A few students did define the expectation value as the probability of measuring a given value [e.g., $-(60 / 68)(\hbar / 2)$ would mean a $60 / 68$ probability of measuring $\hbar / 2]$. In both the spin and energy expectation values problems, student concept definitions related to ensemble average and weighted average were common whether or not students invoked the summation method. In the context of energy, the use of weighted average ideas supported students in thinking about energy expectation value, as they were able to connect the answer to a histogram and have an interpretation for the meaning of the expectation value.

Although the most probable value concept definition did not appear in the spins or energy contexts interviews, it did appear relatively frequently when students described the expectation value of position. In fact, most probable value was the most common definition that students provided for the expectation value of position, even though the weighted average perspective had been the most common for the energy context in the same set of interviews. Wayne's responses are demonstrative of the shift in perspectives. While he describes the expectation value as the value you would expect in both situations, such a description was easier to connect to most probable value when the expectation value is a possible measurement. When students discussed the expectation value of position, there was a larger variety in definitions and more mixed responses and "not sure" responses indicating that students struggled to articulate a definition.

In many cases in both the survey and in interviews, students began to define the expectation value as the value you'd expect, the value you would measure, or the average you would measure and immediately amended the statement with something contradictory. These responses are suggestive of a restricted concept image of the expectation value. Even the use of the word average in a student's definition of the expectation value did not guarantee a concise definition, correct formula, or calculation. Association between value you would expect and the expectation value may make sense from a linguistic perspective. However, since the "value you expect" has the connotation of being the most probable value, it is a different statistical quantity than the expectation value.

In many interviews there was a disconnect between the description of the expectation value and mathematical calculation. Half of students connecting the expectation value of a spin operator to the relative weight of the probabilities did not identify the summation method as a possible solution pathway. Conversely, other students set up appropriate calculations but struggled to connect the expressions for the expectation value to a definition. Leanne in particular expressed being able to find correct answers without having a clear sense of what the expectation value meant. An incorrect definition led to a contradiction for students like Freya, who correctly used summation but made sense of the expectation value as the probability of a value.

The context of a given problem plays a role in what information is evoked from a students' concept image. Consistent with previous literature, the evoked ideas or definitions can appear differently when different features are more salient $[14,18,19]$. This in itself is not surprising or inherently problematic. However, an instructor wanting to choose an expectation problem for an exam may just pick a problem context, thinking students would approach the task universally. Findings from the interviews, however, reveal that a shift from discrete superpositions to continuous wave functions resulted in some students defining the expectation value differently. When working with a superposition of spin states or energy eigenfunctions, students most often drew on a weighted average perspective. "Most probable value" became the predominant definition for position, even for students setting up the appropriate integral.

Our findings suggest that instruction could benefit students in drawing the connections between a spin and wave function context, if more time is spent reinforcing the definition of the expectation value in terms of continuous observables. This could include a discussion of the wave function in terms of "discrete" positions in order to connect the histogram representation to probabilities of measuring position. Similarly, the summation method could be connected to the integration method by discussing integration as a sum over the possible positions weighted by their probabilities. Such an emphasis would draw on students' work with discrete systems where they more often discussed averages connected to higher probabilities of measurement outcomes.

\section{ACKNOWLEDGMENTS}

This material is based upon work supported by the National Science Foundation Grants No. DUE 1626280, No. 1626594, and No. 1626482.
[1] C. Singh and E. Marshman, Review of student difficulties in upper-level quantum mechanics, Phys. Rev. ST Phys. Educ. Res. 11, 020117 (2015).
[2] G. Zhu and C. Singh, Improving students' understanding of quantum measurement. I. Investigation of difficulties, Phys. Rev. ST Phys. Educ. Res. 8, 010117 (2012). 
[3] H. Sadaghiani and L. Bao, Student difficulties in understanding probability in quantum mechanics, AIP Conf. Proc. 818, 61 (2006).

[4] E. Marshman and C. Singh, Investigating and improving student understanding of the expectation values of observables in quantum mechanics, Eur. J. Phys. 38, 045701 (2017).

[5] C. Singh and E. Marshman, Student difficulties with determining expectation values in quantum mechanics, Proceedings of the 2016 Physics Education Research Conference, Sacramento, CA (AIP, New York, 2016), pp. 320-323.

[6] C. Singh, Student understanding of quantum mechanics, Am. J. Phys. 69, 885 (2001).

[7] C. Singh, Student understanding of quantum mechanics at the beginning of graduate instruction, Am. J. Phys. 76, 277 (2008).

[8] E. Gire and E. Price, Structural features of algebraic quantum notations, Phys. Rev. ST Phys. Educ. Res. 11, 020109 (2015).

[9] M. Wawro, K. Watson, and W. M. Christensen, Metarepresentation competence with linear algebra in quantum mechanics, in Proceedings of the 20th Annual Conference on Research in Undergraduate Mathematics Education, edited by A. Weinberg, J. Rasmussen, J. Rabin, M. Wawro, and S. Brown (MAA, San Diego, 2017), pp. 326-337.

[10] B. R. Wilcox, M. D. Caballero, D. A. Rehn, and S. J. Pollock, Analytic framework for students' use of mathematics in upper-division physics, Phys. Rev. ST Phys. Educ. Res. 9, 020119 (2013).

[11] C. Green and G. Passante, The use of ACER to develop and analyze student responses to expectation value problems, Proceedings of the 2017 Physics Education Research Conference, Cincinnati, OH (AIP, New York, 2017), pp. $152-155$.

[12] B. P. Schermerhorn, G. Passante, H. Sadaghiani, and S. J. Pollock, Exploring student preferences when calculating expectation values using a computational features framework, Phys. Rev. Phys. Educ. Res. 15, 020144 (2019).
[13] H. Sadaghiani, G. Passante, and S. Pollock, Student understanding of quantum mechanical expectation values in two different curricula, Proceedings of the 2018 Physics Education Research Conference, Washington, DC (AIP, New York, 2018).

[14] D. Tall and S. Vinner, Concept image and concept definition in mathematics with particular reference to limits and continuity, Educ. Studies Math. 12, 151 (1981).

[15] D. Griffiths, Introduction of Quantum Mechanics, 2nd ed. (Prentice Hall, Inc., New York, 2005).

[16] D. H. McIntyre, Quantum Mechanics (Pearson Education, Inc., San Francisco, CA, 2012).

[17] Adaptable curricular exercises for quantum mechanics, https://www.physport.org/curricula/ACEQM/.

[18] L. Doughty, E. Mcloughlin, P. V. Kampen, L. Doughty, E. Mcloughlin, and P. V. Kampen, What integration cues, and what cues integration in intermediate electromagnetism, Am. J. Phys. 82, 1093 (2014).

[19] B. P. Schermerhorn and J. Thompson, Student determination of different area elements in upper-division physics, Proceedings of the 2017 Physics Education Research Conference, Cincinnati, OH, (AIP, New York, 2017), pp. 356-359.

[20] L. Bollen, P. V. Kampen, and M. D. Cock, Students' difficulties with vector calculus in electrodynamics, Phys. Rev. ST Phys. Educ. Res. 11, 020129 (2015).

[21] B. Schermerhorn and J. Thompson, Physics students' construction of differential length vectors in an unconventional spherical coordinate system, Phys. Rev. Phys. Educ. Res. 15, 010111 (2019).

[22] F. Marton, Phenomenography - Describing conceptions of the world around us, Instr. Sci. 10, 177 (1981).

[23] J. Guisasola, J. M. Almudí, and J. L. Zubimendi, Difficulties in learning the introductory magnetic field theory in the first years of university, Sci. Educ. 88, 443 (2004).

[24] L. Ortiz, P. Heron, and P. Shaffer, Student understanding of static equilibrium: Predicting and accounting for balancing, Am. J. Phys. 73, 545 (2005). 